rately timed readings without the chronograph; frequent readings of different varying temperatures present no difficulties; and the equal timing of the observations greatly facilitates their subsequent treatment.

The latter point is important in calorimetry. As a rule, most of the observations in calorimetry relate to the cooling correction, and are worked up to get the average temperature for a given period of time. For the irregularly timed data given by null readings, the best method of reduction is to plot and apply a planimeter. But this graphic treatment, very properly recommended as a great saving of labor by those who have had in view the null method data, is clearly much more trouble than the simple addition which alone is required for the data obtained by deflection reading. The observer, therefore, will very soon be repaid for the slight labor needed to fit his installation for deflection reading.

The summary is combined with that of the following paper.

\title{
POTENTIOMETERS FOR THERMOELECTRIC MEASUREMENTS ESPECIALLY IN CALORIMETRY.
}

By WALter P. White.

Received July 20, 1914.

In order to be well adapted for accurate thermoelectric work, a potentiometer should show a precision of o.I microvolt, because this precision, as a rule, can readily be reached in the connections and galvanometer, and to fall short of it in the potentiometer involves a waste of facilities, limiting by so much the efficiency of the thermoelement. There are also other features which, though not essential, are desirable in a potentiometer for thermoelectric purposes. The range, however, may be relatively low, so that such potentiometers, in spite of their high precision, are of relatively low cost. To avoid confusion it may be well to point out that some slidewire instruments, specially designated as "thermoelectric," have little in common with the instruments here considered, and are, in fact, peculiarly undeserving of their name on any grounds, having almost no feature which specially adapts them for thermoelectric work, and several which hinder. These potentiometers, however, like most others, are satisfactory in many cases, and particularly in high temperature measurements, where the sensitiveness, and usually the precision also, of the thermoelement, is far in excess of requirements.

\section{Essentials of the Potentiometer in General.}

A potentiometer is a row of resistances in series, through which a current, the "auxiliary" current, is passed from a constant battery. This current is kept constant by occasionally adjusting it so that the "drop" (potential difference) through a fixed resistance balances a standard cell. Then, since in a simple circuit the drop is proportional to the resistance, 
any desired voltage within the range of the instrument can be obtained by connecting to two points of the circuit, between which lies the appropriate resistance. This is illustrated in Fig. $I$, where $P$ and $Q$ are the points, $P O Q$ the resistance, $B$ the battery. In the actual instrument, the contacts at $P$ and $Q$ are the movable contacts of dial switches or slide wires. To measure an unknown electromotive force, the unknown, $X$, is connected across $P$ and $Q$, which

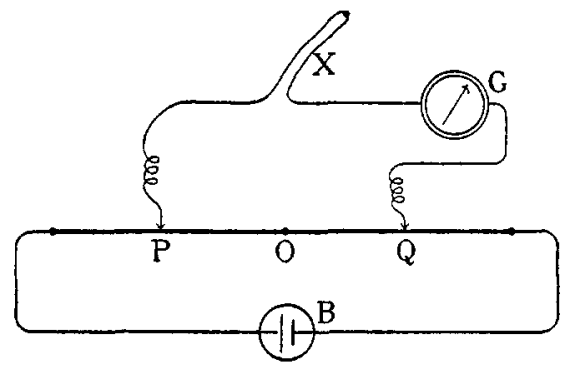
are then moved so as to adjust P O Fig. r.-Potentiometer, simplified. B, bat$Q$ till a galvanometer shows by the absence of any deflection that the unknown is balanced by the voltage tery; $\mathrm{P}$ and $\mathrm{Q}$, contacts; $\mathrm{G}$, galvanometer; $\mathrm{X}$, unknown electromotive force to be measured.

drop of $\mathrm{P} \mathrm{OQ}$, or else, and preferably, an approximate balance is quickly made, and the outstanding difference at once indicated by the galvanometer deflection.

The reasons for the superiority of this second, or "partial deflection," method are given in the preceding paper, Section 9; the necessary condition for it is constant sensitiveness of the galvanometer which, in practice, is an affair mainly of constant resistance in the galvanometer circuit. The galvanometer circuit resistance consists, first, of the thermoelement, galvanometer connections, and ballast, all of which are practically constant in resistance, and second, of the resistance between $P$ and $Q$ in the potentiometer. In "the figure this resistance is the variable resistance $P O Q$ slightly modified by the shunting action of the larger resistance $P B Q$, but in the best potentiometers the total resultant resistance added to the galvanometer circuit by the potentiometer is, by suitable arrangements, made constant, and therefore is usually quite different from the resistance, $\mathrm{P} \mathrm{O}$.

\section{Features Necessary and Desirable for Thermoelectric Work.}

In addition to the obvious and universal requirements of accurate adjustment and adequate insulation, the characteristics desirable iń a potentiometer for thermoelectric work are the following:

(a) There must be no uncertain resistance (such as a contact resistance) in $\mathrm{POQ}$. Even a ten-thousandth of an ohm is usually quite inadmissible.

(b) The movement of the switches must not change the resistance in series with the battery by as much as 0.0000 I of its value, that is, by $0 . I$ ohm or less, as the case may be. Such a change evidently produces a proportional effect on the reading. (In a potentiometer arranged strictly 
as in Fig. I the motion of the switches can not affect this resistance at all, but in some instruments it may.)

(c) The galvanometer circuit resistance should not be changed as much as $0.5 \%$ (that is, as much as one ohm in some cases) by any variation in the resistance $P O Q$, since such a change restricts the use of deflection: reading and therefore hampers work seriously.

(d) The parasitic thermal forces in the galvanometer circuit should be small, and should not be changed by the motion of the switches; that is, the potentiometer should be thermoelectrically neutral. This is much less important for the higher-valued dials than for the lower, since the upper dials are not likely to be so often shifted after the adjustment has been made to eliminate the effect of the parasitic forces. If the potentiometer is not neutral the result is an inconvenience in working, but not necessarily any error.

(e) The resistance should not be too low nor too high. A satisfactory resistance is that which makes the battery current I milliampere. A current as great as twice this begins to cause too great variability in the voltage of a convenient size of storage battery; a resistance three times as high or more may cut down unduly the galvanometer sensitiveness, but may often be desirable.

\section{Types of Potentiometer.}

The attainment of these features is complicated by one requirement not indicated in Fig. $I$, and that is the need for a greater subdivision of the working range than is given by the two switch dials represented there. It is convenient to classify potentiometers first according to the method of making this provision.

(a) By Series Coils.-In one class (Feussner), additional dials are inserted to vary the resistance between $\mathrm{P}$ and $\mathrm{Q}$ (Fig. 2). This arrangement

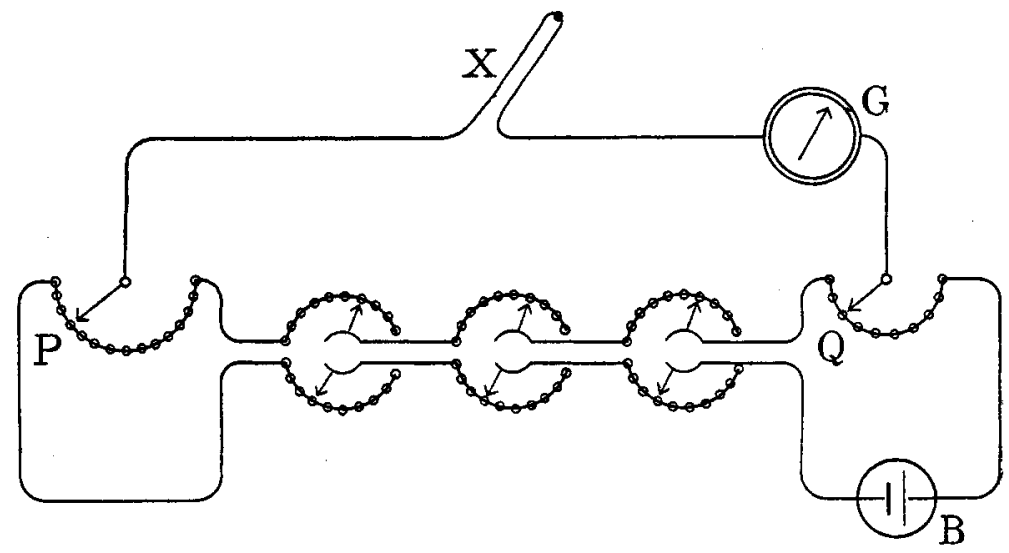

Fig. 2.-General plan of Feussner-Wolff potentiometer. 
is not neutral, and, above all, the switch contact introduces too great uncertainty into $\mathrm{P} O \mathrm{Q}$. The older Wolff potentiometers are of this kind. Although excellently built instruments, they show, unless well cared for, or with a little exposure to hydrogen sulfide, etc., uncertainties of Io microvolts or more, corresponding to a switch contact resistance of o.I ohm. Well cared for, they are reliable to a microvolt, but for other reasons are not very convenient for thermoelectric work, even with this low degree of precision.

(b) By Slide Wire.-In another class of potentiometers one of the contacts represented in Fig. I is upon a slide wire, which, with a dial switch for the other contact, gives sufficient subdivision of the range (Fig. 3). These potentiometers are cheap and widely used. Their proper field is the measurement of rather large voltages by null methods. For accurate thermoelectric work they have several disadvantages: (I) The parasitic forces at the sliding contact frequently reach a large fraction of a microvolt, even in the best instruments. of setting take much longer than in all-switch in-

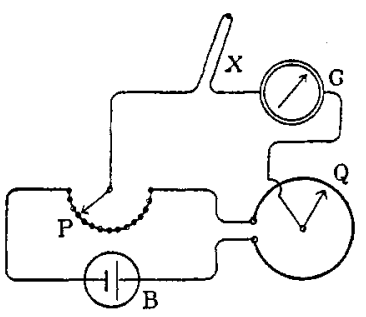
struments, at any rate where deflection reading Fig. 3.-General plan of slide is practiced. This often almost precludes using wire potentiometer. the potentiometer to read more than one thermoelement in the same experiment. (3) The galvanometer sensitiveness is necessarily changed by the motion of the switches, unless a ballast resistance is frequently adjusted to correct the variation. (This adjustment is well worth while if necessary to secure deflection reading, but it is better to get the same advantage without it.) Under slightly unfavorable conditions (such as the presence of even a little corroding gas) or in any but the best instruments, the slide-wire contact resistance is so uncertain as to preclude deflection reading altogether.

These potentiometers, though low-priced for their range, are not, absolutely, as cheap as some lower range instruments of higher precision, and there is therefore seldom any reason for preferring them when purchasing an instrument to use with thermoelements of any description. Instruments already in hand, however, if of the newer and better types, may often be successful, if rapidity is not important, and enough thermoelement sensitiveness can be provided to render sufficient a precision of 0.3 microvolt.

(c) Split Circuit Potentiometers.--Several types of potentiometer provide additional dials by the device illustrated in Fig. 4. Here the contacts $P$ and $Q$ move on two parallel lines of resistances from points $\left(O\right.$ and $\left.O^{\prime}\right)$ originally at the same potential. It is then possible by shunts or external contacts (not shown in Fig. 4) to make changes in the potential of either 
$P$ or $Q$ which are additional to and independent of those produced by shifting $P$ or $Q$, and which do not introduce either contact resistances or thermal

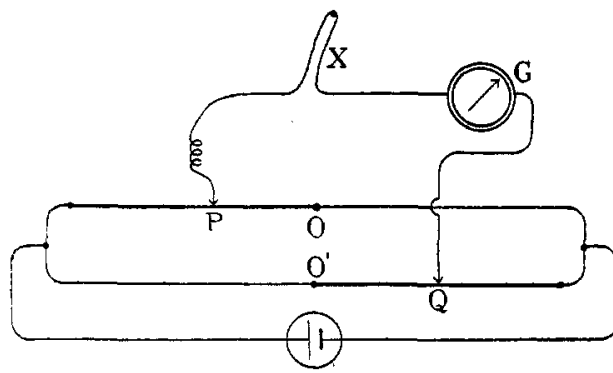

Fig. 4.-General plan of split-circuit potentiometer. forces between $\mathrm{P}$ and $\mathrm{Q}$, nor appreciably alter the galvanometer sensitiveness. Three such devices are elsewhere discussed ; 1 one of them, that invented by Wenner, can be so arranged that no appreciable error will result from a change in contact resistance as great as 0.0003 of the battery circuit resistance, that is, in many cases as great as $0.4 \mathrm{ohm}$. All these arrangements require double switches, that is, dial switches making two separate contacts at the same time.

It appears, then, that in these split circuit potentiometers some of the dials fulfil all the requirements for thermoelectric work enumerated above. For the contacts $\mathrm{P}$ and $\mathrm{Q}$ so completely satisfactory a result can not be obtained, but a choice must be made between very slight evils, which arise as follows: It is possible to have these contacts in the galvanometer circuit, as in Fig. I, or else in the battery circuit as in Fig. 5. The switches will of course have to a slight extent both parasitic forces and resistance. If they are in the galvanometer circuit, the battery current will be completely unaffected by them, and their resistance will therefore be of no importance, so that very simple switches can be used, but their slight thermal forces will be effective, and the potentiometer will not be completely neutral unless they are neu- Fig. 5, - Potentiometer with neutrally-artral. If the contacts are in the bat-

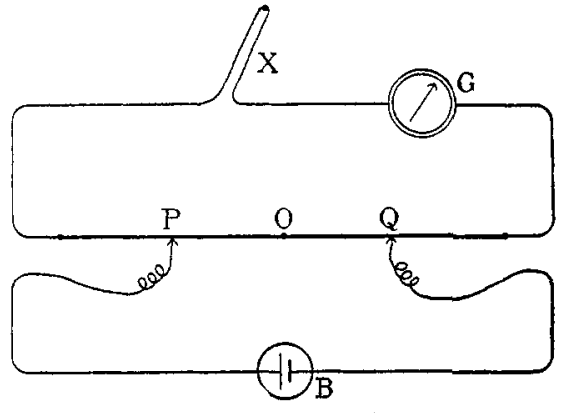

ranged ("antithermoelectric") "contacts. tery circuit, the galvanometer circuit will be quite unaffected and the slight thermal forces will be swamped by the battery voltage, so that the potentiometer will be neutral, but the contact resistances will affect the battery current, and excellent switches of very low resistance will be needed to keep that sufficiently constant. Either arrangement is satisfactory as a rule; neither the resistance nor the thermal electromotive force of a suitable switch is large enough ordinarily to cause appreciable error in these two dials. For reading to 0.01 microvolt the absolutely neutral arrange-

1 "Einige Neue Doppelkompensatoren," Walter P. White, Z. Instr., 34, III-II3, $142-6$ (1914). 
ment, with switches in the battery circuit, seems preferable; the other is certainly preferable if there is any liability of injury to the contacts from corrosive gases. It is worth noting, that as to the thermal forces the eliminating switch ${ }^{1}$ takes care of them, and also renders it impossible for the observer to be in doubt as to their presence or absence, while errors due to contact resistance, on the other hand, are not eliminated or compensated, and are not likely to be even discovered unless specially looked for. This means, however, not that the potentiometer with interrupted battery circuit has any considerable chance of unsuspected error when properly cared for, but rather that the other is "fool proof" to an unusual degree. The advantages of both arrangements are substantially secured by putting the contacts in the galvanometer circuit, and making them neutral. ${ }^{2}$ A potentiometer upon this principle is now in use in this laboratory, ${ }^{2}$ but is at present not regularly on the market. Split-circuit potentiometers of the interrupted battery circuit type ${ }^{3}$ are made by Wolff of Berlin, according to a design by Diesselhorst. One of them, a threedial instrument, can be recommended for thermoelectric work, and is very low in price.

The galvanometer circuit resistance in this Wolff-Diesselhorst instrument is ordinarily about $\mathrm{I} 4 \mathrm{ohms}$. It will be advantageous in most cases to order the instrument made with a resistance ten times as great. ${ }^{4}$ In the galvanometer circuit the increase will merely go to displace some of the ballast necessary to make up the desirable 300 to rooo ohms (see the preceding paper, Section 7), while the battery will be steadier and the possible switch contact resistance error ${ }^{5}$ will be diminished.

The slight, easily avoidable, switch contact resistance difficulties encountered in potentiometers such as that just discussed should by no means be confused with those inherent in resistance measurements. The allowable resistance in a switch of the combination potentiometer, next to be described, is from 3 to ro ohms; in the Diesselhorst potentiometer it is about $0.01 \mathrm{ohm}$, raised to about $0.1 \mathrm{ohm}$ by the increase in total resistance just recommended, while with many resistance thermometers it must be as low as o.0000 ohms, for the same thermometric precision.

The constancy of the galvanometer circuit resistance is not appreciably affected by changes of the contacts $P$ and $Q$ in the Diesselhorst potenti-

${ }^{2}$ Described in the preceding paper, Section 5.

${ }^{2} Z$. Instr. (Loc. cit.), pp. $75^{-78}$, I08-113, I49-150.

${ }^{3} Z$. Instr., 28, I (1908).

${ }^{4}$ This will cause no increase in cost, as Herr Wolff informs me.

5 The order of magnitude of this error can be found in this particular instrument by noting the change in the auxiliary current (as measured against the standard cadmium cell) which is produced by shifting the switches of the upper two dials. Those versed in electrical measurements can also easily find the error of the switches by measuring the potential drop through the contacts. 
ometer, since these dials are like Fig. 5. In instruments where $P$ and $Q$ are like Fig. I, auxiliary coils (not shown in Fig. I) should be added to compensate for the changes produced by moving $\mathrm{P}$ or $\mathrm{Q}$, as is done in several of the Geophysical Laboratory potentiometers. ${ }^{1}$ These coils do not complicate in any way the single contact switches, and they make the total number of coils the same as in the other type, since in that type similar (but more accurate) coils are needed to keep the battery circuit resistance constant.

(d) The Combination Potentiometer. -A fourth method ${ }^{2}$ of adding dials, and one well suited to thermoelectric work, is to connect two otherwise separate $^{3}$ potentiometers in the same galvanometer circuit (Fig. 6). Four

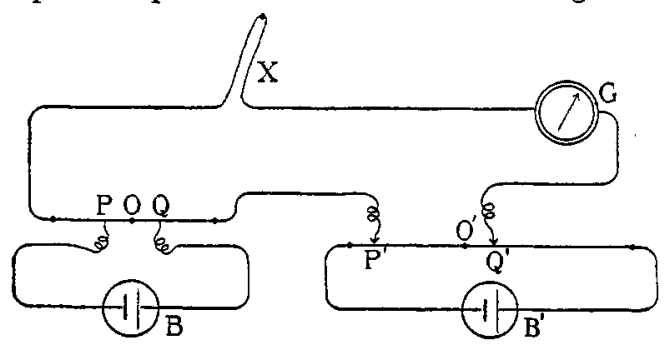
dials in all can thus be obtained, which is perhaps the most advantageous number for a low range. The potentiometer giving the two lower dials may, without detriment to the galvanometer sensitiveness, be given a battery circuit resist-

Fig. 6.-General plan of 4-dial combination poten- ance of 20,000 ohms or more. tiometer. Owing to an oversight, the primes These dials may therefore be of here apply to the upper two dials, not the lower, the type of Fig. 5 without any as in Fig. Ir.

danger of uncertain battery circuit resistance, so that the lower dials are far freer from both resistance and thermal e. $m$. f. troubles than are those of a split-circuit potentiometer. The upper potentiometer may be of either type, just as with the splitcircuit instrument, but the more reliable uninterrupted battery circuit arrangement seems best. Two batteries are required, which is an obvious complication. This difficulty, however, as experience has shown, is distinctly of trifling account, and is offset by several advantages, among which are the simplicity and intelligibility of the electrical arrangement, the ability to check the values of the coils at any time by working one potentiometer against the other, and the fact that all the switches are single. The instrument as shown here is also far safer from contact resistance error than any low-range instrument on the market at present. The mechanical and electrical simplicity of this design render it an easy one to get built to order, and thus recommend it where any special features are desired, such as independence of switch contact resistance, or pro-

I "Potentiometer Installation," Loc. cit., p. 349; Z. Instr., 27, 216 (1907); 34, 108-9 (1914) (Loc. cit.).

${ }^{2}$ Suggested but condemned by H. Hausrath, Ann. Phys., I7, 740 (1905). Described in $Z$. Instr., 34, 147-148 (1914) (Loc. cit.).

${ }^{3}$ The two potentiometers, however, easily may be, and preferably should be, balanced by the same standard cell. Of course they may also be in the same box. 
vision for the double potentiometer arrangement described in Section 5 below. For a double potentiometer this design has another advantage, since the single switches make it especially suitable for the very inexpensive master switch arrangement described in Section 6 .

(e) Choice of Instrument.-It follows from the above that there are three potentiometer types which are thoroughly satisfactory for thermoelement work of precision: the split-circuit type with uninterrupted battery circuit (Geophysical Laboratory), the combination potentiometer (Fig. 6) and the Diesselhorst potentiometer when made with tenfold resistance. Of these the first is not regularly manufactured at present. The combination potentiometer, though not yet regularly listed, is easy to procure, on account of its simplicity, and the Diesselhorst is a stock article. Of the two, the combination potentiometer requires a very little more routine manipulation, but requires less care, and is preferable."

\section{Special Switch Connections.}

(a) When in making the adjustment for eliminating parasitic electromotive forces, the switch of Fig. I in the preceding paper is thrown, interrupting the battery current through the potentiometer, it is still often necessary that a battery current of about the same amount should flow somewhere, for unless the current is very small indeed, 0.0003 ampere or less, its discontinuance will generally cause a perceptible variation in the voltage. The necessary substitute current is readily provided by connecting a suitable resistance between the other end of the battery and the clip A in Fig. $x$.

A new and better way for low-range potentiometers is to connect the clip $A$ to a point between the two potentiometer terminals, so located that the current will still flow much as before through most of the circuit, but will pass around the coils used to balance the unknown, the only ones in which the current needs to be interrupted. To do this the regular connection from the switch runs to the regular potentiometer terminal nearest in potential to the coils in question; and the connection from A runs to a dial contact block just the other side of them. This block thus becomes an "intermediate terminal," I of Figs. 8 and II. The proper location of each terminal can be found by potential measurements upon the potentiometer top, or by tracing the circuit underneath it. ${ }^{2}$ In split-circuit potentiometers the whole split portion must be cut out.

The advantages of the method are that it dispenses with the substitute resistance, and that it avoids at all times the existence in the switch of

${ }_{1}$ Recent quotations, by an American manufacturer, on single and duplicate combination potentiometers, similar to that in Fig. II, make that type, as it should be, the lowest priced of the potentiometers here considered (Aug. I4, I9I4).

${ }^{2} \mathrm{~A}$ binding post connected to the intermediate terminal may be provided, but is not necessary. 
large potential differences, and thus dispenses with the shielding arrangements often needed to prevent leakage errors due to these potential differences. ${ }^{1}$ This advantage holds even where the circuit resistance is so high that interruption of the current causes no unsteadiness, for the danger from leakage increases with the circuit resistance.

(b) Most potentiometers have a key in the galvanometer circuit, often with several"associated safety resistances to guard against excessive galva-

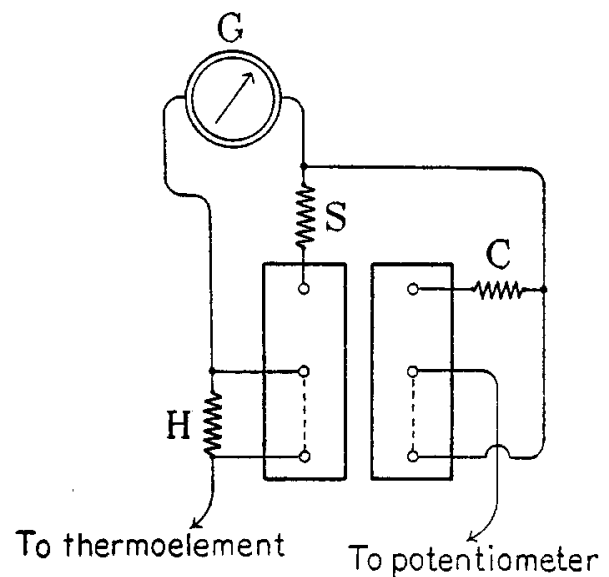

Fig. 7.-Improved galvanometer switches. nometer deflections. This combination is with moving coil.galvanometers less convenient than the arrangement of Fig. 7 , which can be made, at nominal cost, from a couple of single-pole, double-throw switches ${ }^{2}$ and three resistances. The resistance of the coil $\mathrm{H}$ is 9 times that of the rest of the galvanometer circuit -that is, 9 times that in the potentiometer, thermoelement, and associated resistances- $s$ is $\mathrm{ro} / 8 \mathrm{r}$ of $\mathrm{H}$. $\mathrm{C}$ is a very high resistance, IOO,000 ohms or so, of graphite on glass.

When the switch $\mathrm{A}$ is closed at the back end, the galvanometer is shunted so as to be properly damped for all positions of the switch B. With B open there is no deflection. Closing B at the back end gives a very small deflection, due to current through the safety resistance C. Bringing $B$ forward again closes the circuit without $C$, giving exactly one-tenth the full sensitiveness. Bringing $A$ forward disconnects $S$ and short-circuits $\mathrm{H}$, giving full sensitiveness. The usual practice will be, in starting an observation, to close B for I-Io sensitiveness, then set the dial switches, then throw $A$ forward. On opening the circuit both $A$ and $B$ are thrown back together by a single motion. Continuous readings with I-Io sensitiveness are, of course, often very convenient.

This arrangement is discussed in $Z$. Instr., 34, 78-82 (I9I4); (loc. cit.), where those interested can find further details. It is, of course, a privilege and not a requirement in potentiometer work, and it is therefore, for simplicity, not represented in Figs. 8 and I I below. If used it would be between the galvanometer and the point marked $T$.

1 These shielding arrangements will be described in a subsequent paper.

2 The single-pole switches with short throw are advantageous. They are a regular stock article. 


\section{Multiple Reading Arrangements.}

One virtue of electricity in all its applications is the ease with which its power may be applied by the mere touching of two conductors, and the consequent facility with which different apparatus may be combined, or used in rapid succession. Among measuring devices of precision the potentiometer-thermoelement combination possesses this facility in a high degree, owing to its indifference to contact resistance outside the potentiometer (and often within it); to its adaptability to the partial deflection method, which favors rapid and varied measurements; to the quickness and ease with which equal and interchangeable thermoelements can be constructed; and to the power which the thermoelement has of giving readings with the galvanometer alone. Simultaneous measurements, that is, quickly alternating observations, of the temperature of different bodies or regions are sometimes essential and often recognized to be desirable, and would be found so still more often if convenient means for making them were at hand.

With provision for getting the last two figures of the reading from the galvanometer deflection it will usually be satisfactory to make successive measurements of different temperatures by resetting the switches; there is nearly always ample time for this in calorimetry. ${ }^{1}$

Usually, also, some of the quantities measured are, or can be made to be, small enough to fall within the limits of the galvanometer scale; provision for reading these by the galvanometer deflection alone can be made by a slight addition to the auxiliary switch system, and much resetting of the switches thus avoided. Fig. 8 shows one arrangement for this purpose,

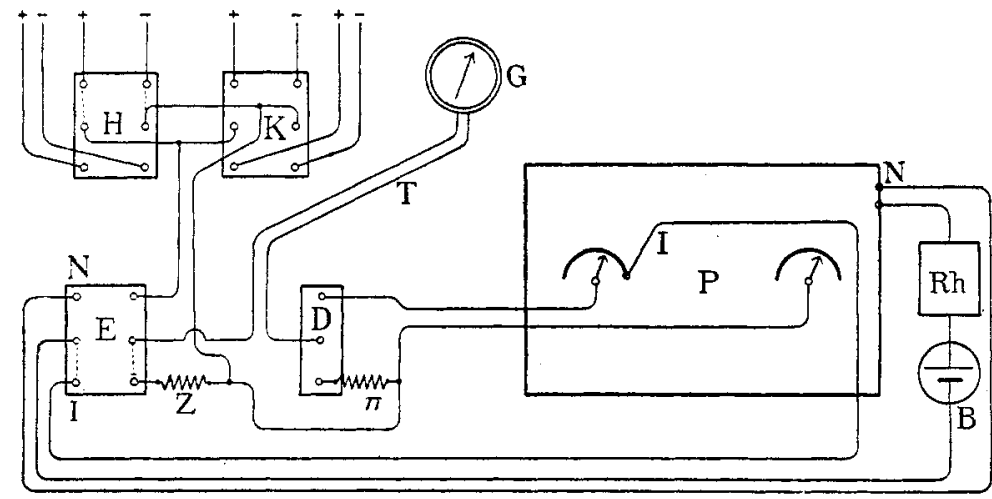

Fig. 8.-Arrangement for combined direct and potentiometer reading of 4 thermoelements. $P$, potentiometer; $I$, intermediate terminal; $N$, negative terminal; $R h$, rheostat; $\mathrm{B}$, battery; $Z, \pi$, substitute resistances; $\mathrm{H}, \mathrm{K}$, thermoelement exchanging switches; E, eliminating switch; D, potentiometer-cut-out, or direct-reading, switch; $T$, location of galvanometer and cadmium cell switches.

1 This remark may not apply to slide wire instruments. 
operated by means of the double-throw knife switch D. Any one of the thermoelements (here 4 in number) can be instantly connected, either to the potentiometer, or else to the substitute resistance $\Pi$, which is equal to

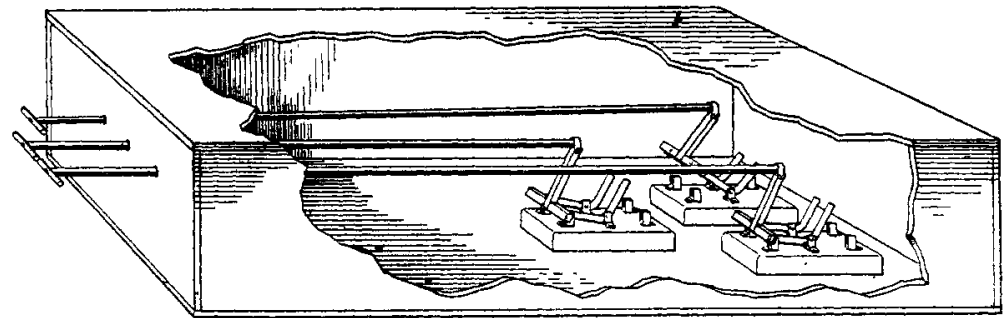

Fig. 9.-Perspective of switches, showing double-ended knives, pull-rod connections, and method of working two switches together.

the constant galvanometer circuit resistance in the potentiometer. $\mathrm{E}$ is the eliminating switch of Fig. I in the preceding paper, here used with "intermediate" battery circuit connection, I, as described above in Section 4.

Fig. 9 shows a construction which favors the rapid operation of the other switches. Without it, a resetting may involve three motions. First one thermoelement switch is opened, then the potentiometer-cut-out switch D is thrown across, and then the thermoelement switch is closed on the other side. By grasping at once two of the adjacent handles of Fig. 9, the observer can make this, the most common resetting, in one motion, and never need make but two. The thermoelements which are to be used with the far position of $\mathrm{D}$, that is, with the potentiometer in circuit, are then on the far side of the switches $\mathrm{H}$ and $\mathrm{K}$, those to be used with the near position of $\mathrm{D}$ are on the near side of $\mathrm{H}$ and $\mathrm{K}$. This system avoids the chance of an unusual combination's occurring by mistake, but does not destroy the possibility of making it whenever that becomes desirable. There is, however, surprisingly little danger of confusion, with this or any other system. A habit of moving the switches correctly is formed literally in five or ten minutes.

In our switch the pull rods are merely pieces of wooden doweling. They run through guides at the back end also and each is connected to its switch by a pair of connecting rods cut from sheet brass strip. This arrangement works more smoothly and is perhaps a little easier to construct, also, than the one shown, which was chosen for representation as making a more easily intelligible drawing. The handles to the switches are metal bars, slotted so as to fit very tight over the insulating cross bars of the switches and further held by screws. The object of this construction was to make the greatest possible provision against danger of having the handles work loose, which is almost the only way in which this kind of switch can give trouble. If $D$ is to be used.with 4 or more switches like $\mathrm{H}$ and $\mathrm{K}$, its pull rod is best made of steel for stiffness.

The simultaneous motion of the switches $\mathrm{D}$ and $\mathrm{H}$ or $\mathrm{K}$ is made automatic in our switch in the following way: $\mathrm{D}$ has a shorter handle, so that it is thrown by half the pull-rod motion needed by $\mathrm{H}$ or $\mathrm{K}$. On the rod of $\mathrm{D}$ are two cross bars, as far apart as the throw of $\mathrm{D}$ (or half the throw of $\mathrm{H}, \mathrm{K}$, etc.) and so situated as to be pushed by pins passing through the rods of $\mathrm{H}, \mathrm{K}$, etc. Then, whenever any of the thermoelement switches $H, K$, etc., is thrown, it brings $D$ into the right position, leaving the others 
of the $\mathrm{H}, \mathrm{K}$ set in the middle (open) position, and bringing to that open position any thermoelement switch that may have been set on the other side. To make a setting, then, it is only necessary to grasp the handle of the proper thermoelement switch and throw that, when everything else will follow, except in changing from one switch to another on the same side, when two half throws are required. Any unusual combination is readily secured by withdrawing the appropriate pin from its pull rod. It may be well to remark that the less elaborate arrangement shown in the figure is quite satisfactory, but the installation of the more convenient automatic one should not cost an hour's labor, and therefore seems generally worth while.

With the scheme of connections shown in Fig. 8 the usual direct galvanometer connection to the potentiometer must be omitted during regular readings, but restored for the adjustment against the standard cell. The necessary transfer is easily made by a 3-pole switch, as shown in Fig. Io. The switch would be inserted at the point $T$. The galvanometer terminals of the potentiometer are short-circuited by the righthand knife during regular readings, when the galvanometer is in the circuit at another place.

It will often be desirable to read two different and rather large electromotive forces in the same experiment, for which the arrangement just described is of course inadequate.

Resetting of the switches can then usually be facilitated by a simple device. As a rule the observation of two different temperatures will involve making first one setting, then a second, different one, then the first again, then the second, and so on, with comparatively infrequent changes in either setting. But the time and effort of switch manipulation go mainly not in the mechanical operation of moving the switches, but in thinking where to move them and at the same time trying to be To switches sure that no mistake is D \& E

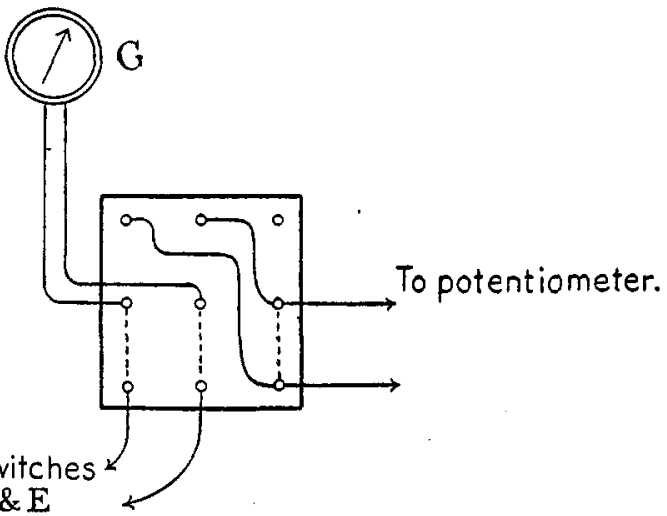
made. Most of the.labor Fig. ro.-Switch for making the cadmium cell connection is therefore saved if the in the arrangement of Fig. 8. Inserted at T, Fig. 8. two alternate settings are limited by stops against which the switch can be quickly thrown without even looking, and which are shifted from time to time as the settings themselves change. Very convenient stops can be made of strips of thin hard rubber, which are inserted between the switch blocks. An indicator appealing to the eye may also be used. The dial switches are moved after one thermoelement switch is opened, and before the other is closed. Three or four seconds is ample time for the whole operation. 
I 880

WALTER P. WHITE.

6. Double Potentiometers.

A more convenient arrangement is to have two sets of switches, and put one or the other into action by a single unvarying motion of a master

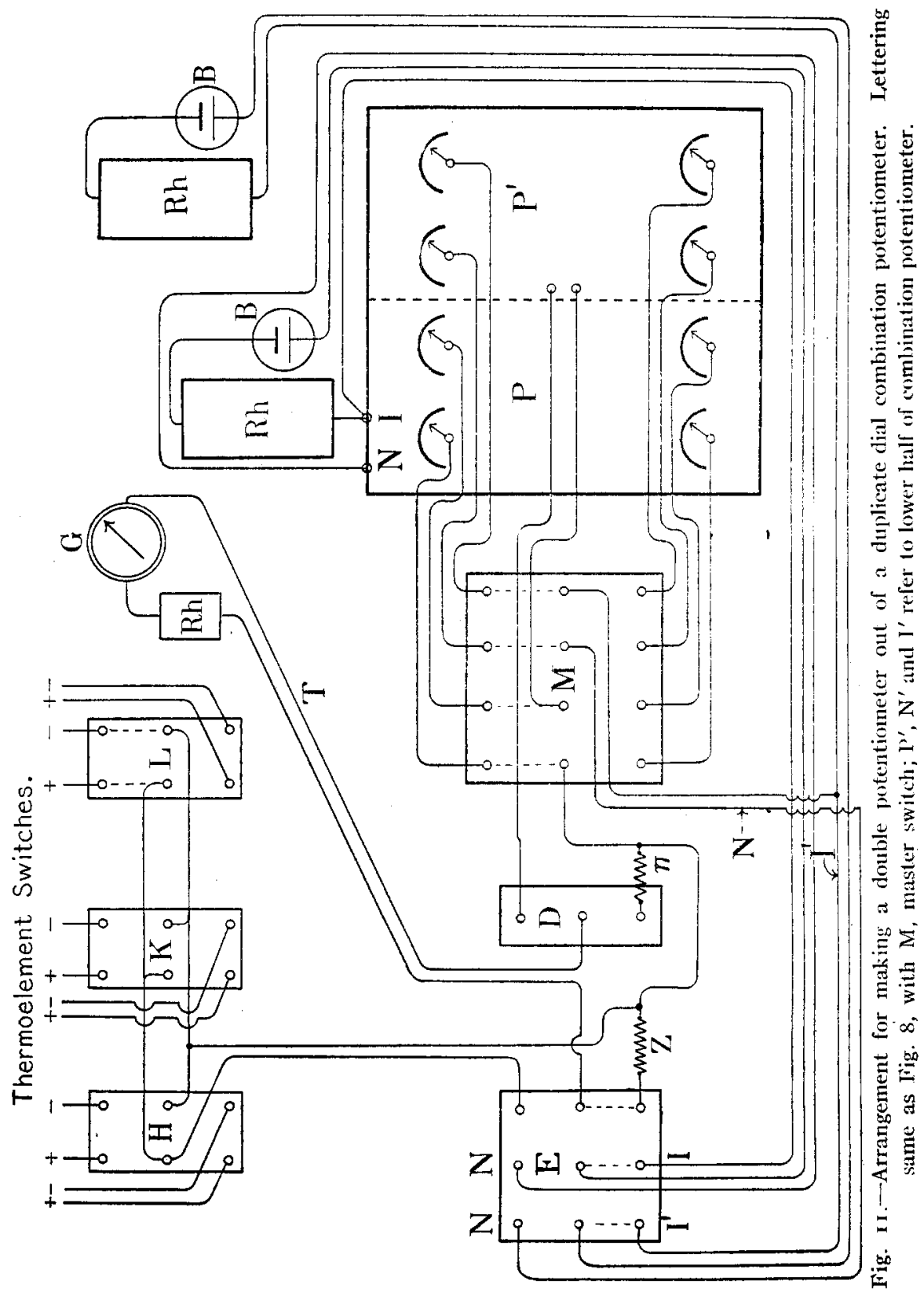


switch. This gives a double potentiometer. ${ }^{1}$ It can, of course, be made up as a complete instrument. In that case, especially if a split circuit instrument is the basis, an adequate master switch which is also compact enough is rather expensive, and is preferably avoided by the gridiron construction already referred to. ${ }^{2}$ But where extreme compactness and conventional finish are disregarded, as they may be if the observer connects his own apparatus, it is very easy indeed, especially with the combination potentiometer of Section 3 (d), above, to arrange a double potentiometer as efficient as the best. The master switch and accessories, made up of various knife switches, are shown as connected to a combination potentiometer in Fig. I r. The arrangement for reading by galvanometer deflection alone is also provided here, by means of the single switch in the middle.

The eliminating and potentiometer-cut-out switches $\mathrm{E}$ and $\mathrm{D}$ are exactly as in Fig. 8, except that $E$ handles 2 batteries, but in place of the simple potentiometer of Fig. 8 occurs the master switch $M$, leading to the combination potentiometer P. M, when thrown, exchanges the upper dial switches in the galvanometer circuit and the lower dial switches in their battery circuit. The battery circuit of the upper dials and the galvanometer circuit connection to the lower dials remain unaltered. $\mathrm{Rh}$ are 3 rheostats for adjusting the two battery circuits and the galvanometer circuit. For simplicity, the intermediate battery eircuit terminals I and $I^{\prime}$ (of Section 4 ) are located as if most of each battery circuit resistance were in $\mathrm{Rh}$; the exact place of the connection in practice will depend on the arrangement of the particular instrument used. The other connections are exactly as they might be in any case, with a combination potentiometer.

The fact that a combination potentiometer is used, with two batteries to adjust, of course makes the cadmium standard cell connection somewhat different from that of Fig. Io. Fig. I2 shows an arrangement calling for 2 ordinary 2-pole, 2-throw knife switches. It is, of course, to be inserted at the point marked "T." The leads at the right run to fixed resistances such as are frequently used in potentiometers for the standard cell adjustment.

This particular arrangement is appropriate to a special instrument, whose galvanomèter connection is intended to be made through the switches $D, E$, and M, with either a key, or a switch like that of Section 4 (b) in the circuit near the galvanometer.

A simple system for operating the switches $\mathrm{D}, \mathrm{M}, \mathrm{H}, \mathrm{K}$, etc., is to have the thermo-

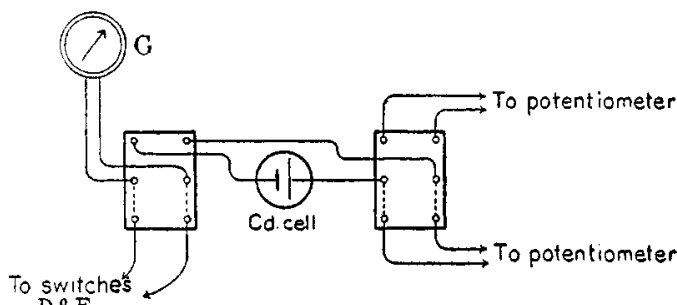
D\&E

Fig. 12.-Cadmium cell switches for combination potentiometer of Fig. I I. Inserted at $T$. element switches at the right-hand end of the line thrown forward and back in unison with $M$, according to the method illustrated in Fig. 9, and the switches at the left

${ }^{1}$ Described and discussed in $Z$. Instr., 34, 73-78 (1914) (Loc. cit.). First proposed, Ibid., 27, 218 (1907). Triple potentiometers are of course possible, but the advantages decrease relatively, and the difficulties increase, as the number of sets of switches increases.

2 Z. Instr., 34, I I I-I 42 (Loc. cit.). 
similarly thrown forward with $\mathrm{D}$, and also thrown back while $\mathrm{D}$ comes forward, using this last, less rapid combination for the least frequent readings.

The arrangement of switches as shown here will be from 20 to $30 \mathrm{~cm}$. wide if "I5. ampere" knife switches of stock type are used. Its depth will depend on the number of switches added to those shown here, and therefore on the number of thermoelements provided for. If pull rods are used, as in Fig. 9, a relatively large number of switches. can easily be accommodated by putting them beyond those shown in the figure. $A$ considerable extension of the switchboard in the direction away from the operator is not objectionable. There is, therefore, as far as table space is concerned, no difficulty in. providing for as many thermoelements as any one is likely to use.

Every essential connection and piece of apparatus has been here indicated. It therefore appears that the advantages of the double potentiometer can be obtained practically at the cost of a few knife switches, for the extra dial switches can be provided at negligible cost in several ways. ${ }^{1}$ If a combination potentiometer is made on special order, a duplicate set: of regular dials will also cost comparatively little.

The master switch here illustrated is, of course, readily applicable to any potentiometer whose switches do not need to maintain a very low contact. resistance, but not as readily to any other. At present no such potentiometer is regularly made which is also suited for thermoelectric work, hence a special instrument, and preferably, for reasons given in Section 4, a combination potentiometer, affords the best means of realizing a doublepotentiometer: This, however, may be only a temporary situation. Meanwhile, those who need only a single potentiometer will generally find the Diesselhorst type satisfactory; those who need either a doublepotentiometer or special immunity from contact resistance error can haveboth, at no great expense, by means of a special instrument of the combination type.

\section{The Number of Dials Required.}

If the galvanometer deflection is utilized, three dials, with a maximum range of I0,000 microvolts (as in some of the newer Diesselhorst-Wolff potentiometers), are quite sufficient for calorimetry. They permit reading to O.I. microvolt, and up to a $10^{\circ}$ interval with a 24-couple thermoelement. For larger temperature intervals a part of the thermoelement can be used, and though the precision is then less absolutely, it will still be of the order of $i$ in 50,000 .

For high temperature work, even with a single couple, readings above 10,000 microvolts but below 20,000 are usually needed. They can be provided for with three dials by doubling the current, but the absolute precision is then reduced to 0.2 microvolt. (The double range can be obtained by putting two standard cells, in series, in place of one in an instrument intended for the single range, or it can be provided for in the original construction by the insertion of a suitable coil.)

Voltages above 20,000 are sometimes desirable. A four-coil instrument with range of 100,000 microvolts will provide for them, and with a somewhat lower range

1 One method which we have had in use for five years is to screw, to the regular dial contact blocks, tongues of sheet copper $0.5 \mathrm{~mm}$. thick and make contact with these by clamps like Fig. 2 of the previous paper. The relative slowness of this kind of a dial has proved to be but a trifling disadvantage in a double potentiometer. 
$(30,000$ to 50,000$)$ and a sufficiently sensitive galvanometer will, at the same time, give an absolute precision in reading of better than 0.1 microvolt, which may be helpful in some cases.

\section{Intrinsic Merits of the Thermoelement-Potentiometer Combination.}

In view of the amount of detail contained in this and the preceding paper, the cursory reader would scarcely be at fault if he should get the impression that thermoelectric apparatus is elaborate and complicated, and its use beset by pitfalls and precautions. It therefore seems worth while to show, briefly, that the exact opposite is the case. The only essentials peculiar to this system are, as already stated, a galvanometer sensitiveness readily attainable, potentiometer excellences which are admirably embodied in two very low-priced designs, and a common copper knife switch, arranged as an eliminating switch with neutral leads. The amount of detail presented is due, first, to the attempt to give what are practically working directions in a generally unfamiliar subject, and second, to the presentation of arrangements for securing in the observations a rapidity, convenience, and comprehensiveness such as are seldom even thought of in most other systems. The net final effectiveness attained can be fairly well seen by a comparison with the calorimetric resistance thermometer, which has come to be widely recognized as an accurate and convenient instrument.

(a) The relative precision required in the electrical measurement is more than a hundred times less with the thermoelement.

(b) The thermoelement is well known to be far less affected by defects of insulation.

(c) Parasitic thermal electromotive forces, the only other noticeable sources of error, require, for their elimination, an arrangement which, though a little more extensive in the case of the thermoelement, is quite as rapid and effective in operation.

(d) The complications and errors arising from the resistance of contacts and leads, so prominent with the resistance thermometer, have been eliminated with the thermoelement.

(e) Very rapid readings and readings made at definite instants.of time are more easily secured with the thermoelement system.

$(f)$ The same is true of simultaneous readings of different temperatures, and of concomitant measurements of electrical energy.

(g) The constancy or integrity of the multiple thermoelement can be quickly tested at any time, without interrupting or disturbing any determination that may be in progress. A corresponding test with the resistance thermometer necessitates placing it in a carefully prepared ice bath.

(h) No correction for heat developed, or precautions to avoid undue heat production, are necessary with the thermoelement. 
The thermoelement has the disadvantage that some body of definite thermal condition must surround the end not in the calorimeter. This necessity, which will be considered in a later paper, adds visibly to the apparatus required and slightly to the daily manipulation, practically not at all to the chances of error. But on the electrical side the calorimetric thermoelement appears superior in several respects, inferior in none ${ }^{1}$ of importance.

\section{Summary of this and the Preceding Article.}

These two papers describe a type of auxiliary installation for thermoelements which in high temperature measurement and other work of moderate precision is valuable for its convenience, quickness, and comprehensiveness, and which is also capable of the very high precision often desired for calorimetry.

(I) When a thermoelement is used with its two ends at nearly the same temperature, a condition easily provided in calorimetry, the relative precision required in the electrical measurement falls to a value no greater than that desired in the temperature reading, and the most serious errors ordinarily affecting the electrical thermometer practically disappear.

(2) The absolute electrical precision required is also comparatively low. With a convenient and easily made copper-constantan multiple thermoelement of 24 couples, $0.0001^{\circ}$ corresponds to o.I microvolt.

(3) The satisfactory attainment of a precision of 0.1 microvolt demands two, and only two, special electrical instruments. The first is an arrangement for eliminating the effect of parasitic thermal electromotive forces. A common copper knife switch will perform this service admirably.

(4) The second special requirement is an appropriate potentiometer, that is, one reliable to o.r microvolt.

(5) The slide wire and Feussner potentiometers of I volt range or more now in common use are not thus reliable, and are otherwise unsuited for thermoelement work. Split-circuit potentiometers are satisfactory in this and all other important respects, and so are combination potentiometers, or potentiometers having two otherwise separate, very simple instruments in series in the same galvanometer circuit. A very low-priced split-circuit potentiometer is on the market; and the combination potentiometer, on account of its mechanical and electrical simplicity, is an easy instrument to build to order.

(6) The potentiometer system, either with or without the thermoelement, is especially suited to simultaneous measurements of different and differently varying electromotive forces. Its convenience for such measurements can be increased by using a few pieces of hard rubber sheet as stops for the dial switches, and still further increased by arranging a

1 This statement and some of the discussion immediately preceding, applies, of course, mainly to the measurement of small temperature intervals, as in calorimetry 
double potentiometer, with duplicate dials. One effective form of double potentiometer, which employs a master switch, can be arranged at the cost of a few knife switches and very little labor. An especially suitable instrument to arrange in this way is the combination potentiometer, all of whose dial switches are single, and free from contact resistance error.

(7) Another convenience especially easy to obtain with the thermoelement-potentiometer system is the power to take the last two figures of any reading directly from the galvanometer scale. It increases speed, simplifies manipulation, diminishes errors, and gives calorimetric data in a form specially convenient for further treatment.

(8) For high temperature measurements and much other thermoelement work not calorimetric, though the required precision may often be less, most of the features above described are desirable, especially the facility for simultaneous and direct readings.

GEOPHYSICAI LABORATORY,

CARNEGIE INSTITUTION OF WASHINGTON.

[CONTRIBUTION FROM THE CHEMICAL LABORATORY OF THE STATE UNIVERSITY OF KENTUCKY.]

\section{A STUDY OF THE REACTION OF ALKALI SALTS OF SULFONIC ACIDS WITH ALKALI PHENOLATES BY DRY DISTILLATION.}

By Edgar H. Nollau and lloyd C. Danifis.

Received June 11, 1914.

By analogy to the reaction of alkali sulfonates with alkali hydroxides in the preparation of phenols, aromatic ethers should result from the distillation of sulfonates with phenolates. Results confirming this idea having been obtained with the simplest mixture of the kind, viz., sodium benzenesulfonate and potassium phenolate, a study of the reaction was undertaken with a view to determining whether it is generally applicable. The trend of the proposed investigation is exhibited by the following mixtures that have been used:

I. Sodium benzenesulfonate and potassium phenolate.

2. Potassium toluenesulfonate and sodium phenolate.

3. Sodium xylenesulfonate and potassium phenolate.

4. Sodium sulfanilate and potassium phenolate.

5. Sodium toluidinesulfonate and potassium phenolate.

6. Sodium $\alpha$-naphthalenesulfonate and potassium phenolate.

7. Sodium $\beta$-naphthalenesulfonate and potassium phenolate.

8. Sodium benzenesulfonate and potassium thymolate.

9. Sodium benzenesulfonate and mono-potassium resorcinate.

ro. Sodium benzenesulfonate and di-potassium resorcinate.

I I. Sodium methylsulfonate and potassium phenolate.

The problem was to find what radicals might be represented by $R$ 